\title{
Prediction of flow effects in quantitative NMR measurements
}

\author{
Anne Friebel, Thomas Specht, Erik von Harbou*, Kerstin Münnemann, \\ Hans Hasse \\ Laboratory of Engineering Thermodynamics (LTD), University of Kaiserslautern, \\ Germany
}

\begin{abstract}
A method for the prediction of the magnetization in flow NMR experiments is presented, which can be applied to mixtures. It enables a quantitative evaluation of NMR spectra of flowing liquid samples even in cases in which the magnetization is limited by the flow. A transport model of the nuclei's magnetization, which is based on the Bloch-equations, is introduced into a computational fluid dynamics (CFD) code. This code predicts the velocity field and relative magnetization of different nuclei for any chosen flow cell geometry, fluid and flow rate. The prediction of relative magnetization is used to correct the observed reduction of signal intensity caused by incomplete premagnetization in fast flowing liquids. By means of the model, quantitative NMR measurements at high flow rates are possible. The method is predictive and enables calculating correction factors for any flow cell design and operating condition based on simple static $T_{1}$ time measurements. This makes time-consuming calibration measurements for assessing the influence
\end{abstract}

*Corresponding author: Erik von Harbou (erik.vonharbou@mv.uni-kl.de), presently with BASF, SE, reaction technology 
of flow effects obsolete, which otherwise would have to be carried out for each studied condition. The new method is especially interesting for flow measurements with compact medium field NMR spectrometers, which have small premagnetization volumes. In the present work, experiments with three different flow cells in a medium field NMR spectrometer were carried out. Acetonitrile, water, and mixtures of these components were used as model fluids. The experimental results for the magnetization were compared to the predictions from the CFD model and good agreement was observed.

Keywords: quantitative flow NMR spectroscopy, modeling of magnetization, Bloch-equations, computational fluid dynamics, flow cell, Benchtop NMR spectrometer

\section{Introduction}

Nuclear Magnetic Resonance (NMR) spectroscopy is a widely used analytical technique to elucidate molecular structures and determine compositions of complex multicomponent systems. Quantitative flow NMR spectroscopy is becoming increasingly important for reaction monitoring [1-4] and process control $[5,6]$. The compact medium field NMR spectrometers, that have become available commercially in the recent years, expand the field of application of NMR spectroscopy in these domains considerably. The most common approach in flow NMR spectroscopy is to connect the investigated process with the spectrometer by a sample loop $[1,7,8]$. Also other techniques for NMR spectroscopic reaction and process monitoring have been described in the literature [9-17]. All these applications benefit from the non-invasive characteristics of NMR spectroscopy making it a powerful tool 
for online monitoring.

However, the quality of NMR data depends on the flow conditions in feed line and flow cell, where the measurement takes place. The build-up of the magnetization plays a central role in here. It depends on the residence time distribution (RTD) of the sample in the premagnetization zone of the feed line and the arrangement of that zone in the magnet. A sufficiently long residence time is important to reach the equilibrium magnetization of the nuclei before they are excited in the radio frequency coil. A lack of premagnetization leads to a reduction of signal intensity in the spectrum. The decrease depends on the flow conditions and the longitudinal relaxation time $T_{1}$ of the studied nucleus. In case of insufficient premagnetization, the observed peak intensity is no longer proportional to the mole ratio of the nuclei in the sample, such that quantification becomes difficult. Premagnetization is a particularly important issue in flow measurements with compact medium field NMR spectrometers because of the small size of the magnet and the resulting small premagnetization volume [18].

The need for sufficient premagnetization leads to a upper limit of the flow rate that can be applied in a quantitative NMR flow measurement. This limitation often hurts, as high flow rates in the lines between the process and the NMR spectrometer are desired in order to decrease the time delay between the investigated process and its monitoring. Various measures to overcome this problem have been proposed in literature: application of split bypasses [1], flow cells with expansions in the NMR active region [19, 20] and, an increase of premagnetization by prior external magnetic fields or loopy flow cells [21-25]. All these options have drawbacks when applied in 
reaction and process monitoring, like an increased time delay or increased complexity of the experimental setup.

A correction for the decrease of the signal intensity due to incomplete premagnetization is an attractive option to solve the premagnetization issue. Such a correction term, which is a function of the flow rate and the $T_{1}$ relaxation time has been established by Arnold and Burkhart [26]. These authors describe a model in which the Bloch-equations are coupled to a transport equation for laminar and plug flow in capillaries assuming an external magnetic field with rectangular shape. The analytical solution of this problem was used by Dalitz et al. [27] to quantitatively evaluate NMR spectra acquired online with high flow rates. However, only pure substances were considered in [27]. Furthermore, the model of Arnold and Burkhart [26] is only appropriate for capillaries with constant diameter and the correction is time-consuming as signal intensities in dependence of flow rate have to be measured for all pure components as well as for all mixtures investigated in the process.

For complex flow cell geometries and for a more general consideration, a model is needed that predicts the velocity profile and the RTD in an arbitrary flow geometry. Based on this, the magnetization of an arbitrary component (characterized by: self-diffusion, viscosity, $T_{1}$ relaxation time) in an arbitrary NMR spectrometer (characterized by: premagnetization volume, NMR active volume, shape of external magnetic field $B_{0}$, geometry of flow cell) can be predicted by the model. The most appropriate method for this application is computational fluid dynamics (CFD), which solves the Navier-Stokes equations for a given geometry. As the flow is independent of the magne- 
tization, the transport equations of magnetization can be introduced into the CFD model in a straightforward manner as an add-on. Much work has been done for characterizing velocity fields in NMR flow cells by means of CFD simulations $[19,28,29]$ and a first report is available on work in which the transport equations of magnetization were introduced in the CFD model to qualitatively visualize the magnetization of fluids in NMR flow cells [30]. However, only pure liquids were considered and no quantitative comparison of experimental and simulation data of the magnetization was provided. Diffusion effects were considered, but for numerical reasons diffusion coefficients were chosen that were much higher than those observed in real liquids [30].

In the present work, we discuss in detail how the Bloch-equations can be implemented into a CFD code. The model of the external magnetic field is based on measurements of the magnetic field strength in a medium field NMR spectrometer. We then describe results of the application of the augmented CFD code for predicting the component- and flow rate-specific signal reduction due to incomplete premagnetization. The predictions are tested by a comparison to experimental data and it is shown how ${ }^{1} \mathrm{H}$ NMR data measured at high flow rates can be corrected such that quantitative results are obtained. In this work, acetonitrile and water as well as mixtures of these components were used as model fluids. These components were chosen, as their mixtures show a strongly non-linear behavior of the $T_{1}$ relaxation time as a function of the composition, which makes them a demanding test case. The relative magnetization in three different flow cells was predicted and compared to experimental data. Compositions of mixtures that are measured at high flow rates were corrected by means of the relative magnetization 
obtained from simulation. The simulation results agree well with the experimental data. This shows that the method presented here is appropriate to acquire quantitative NMR data from fast flowing samples in multicomponent systems and complex flow cell geometries. The method is easily applicable to other flow cell geometries and tedious calibration measurements with a limited application range are no longer necessary.

\section{Experiments}

\subsection{Chemicals}

Water $(\mathrm{W})$, acetonitrile $(\mathrm{ACN})$, and mixtures of these components were used as test fluids. Ultrapure water was produced using a Milli-Q integral water purification system from Merck Millipore. Acetonitrile was supplied from Carl Roth with the grade Rotisolv (Purity $\geq 0.999 \mathrm{~g} / \mathrm{g}$ ).

\subsection{Experimental setup}

The dependence of the relative magnetization of nuclei upon the flow rate was investigated in a flow setup.

The flow setup consists of a feed line (PEEK capillary with $d_{\mathrm{i}}=1 \mathrm{~mm}$ ) connected to the flow cell which is positioned in the medium field NMR spectrometer (Magritek Spinsolve ${ }^{\circledR} 1$ T Carbon NMR spectrometer). The liquid is taken from a storage vessel and is pumped through the feed line by a high-pressure dosage pump (Wadose Plus, Wagner MSR). The volume and mass flow rate and the temperature were determined with a Coriolis flow sensor (Mini Cori-Flow, Bronkhorst, Accuracy: $\pm 0.2 \%$ of measured value). The internal temperature sensor has an accuracy of $\pm 0.5 \mathrm{~K}$. 
Three types of flow cells were used, which are shown in Figure 1: flow cell I with constant inner diameter (PEEK, $d_{\mathrm{i}}=1 \mathrm{~mm}$ ), flow cell II with linear expanding inner diameter (glass, $d_{\mathrm{i}}$ from $0.5 \mathrm{~mm}$ to $2 \mathrm{~mm}$ ), flow cell III with step function of inner diameter (PEEK, $d_{\mathrm{i}}$ from $0.25 \mathrm{~mm}$ to $1 \mathrm{~mm}$ ). Figure 1 shows the location of the flow cells in the NMR spectrometer in relation to the location of the radio frequency coil and the overall magnetic field strength $B_{0, z}$. The $x$-axis represents the height inside the bore of the magnet. $x=0 \mathrm{~m}$ is located in the lower, $x=0.3 \mathrm{~m}$ in the upper part of the spectrometer. The external $B_{0, z}$ field was investigated in preliminary experiments with an hall effect magnetometer (see Supplementary Material for detailed description).

\subsection{Procedure}

\subsubsection{Physical properties}

The density of mixtures of acetonitrile and water was measured with a density meter (DMA5000, Anton Paar, Accuracy: $\pm 7 \cdot 10^{-6} \mathrm{~g} / \mathrm{cm}^{3}$ ) at 298.15 K. Before measurement, a calibration with water was performed. Each sample was degassed for $5 \mathrm{~min}$ in an ultrasonic bath prior to measurement. The measurement was repeated at least once.

The $T_{1}$ relaxation time of mixtures of acetonitrile and water was measured with a standard inversion recovery sequence (number of scans: 2, acquisition time: $6.4 \mathrm{~s}$, repetition time: $30 \mathrm{~s}$, max. inversion: $10 \mathrm{~s}$, number of steps: 41) with the Spinsolve ${ }^{\circledR}$ NMR spectrometer in standard $5 \mathrm{~mm}$ Wilmad NMR glass tubes at $302 \mathrm{~K}$. Prior to integration, post processing of the obtained NMR spectra was carried out with the MNova software (MestReLabs). For the baseline correction, a Whittaker smoother algorithm 
was used. The phase correction was done manually. All peaks were integrated without deconvolution as the evaluated peaks were well separated. The $T_{1}$ relaxation time was obtained using the regression procedure provided by the MNova software. Each measurement was repeated at least once, those with pure components were repeated five times. For the latter, the standard deviation was $\pm 3 \%$; this is also the typical deviation between the experimental results in those cases, where only two experiments were carried out. Considering also errors caused by post processing (e.g. manual phasing), the relative uncertainty of the $T_{1}$ measurements is estimated here to be $\pm 5 \%$. A correlation for describing the temperature-dependence of the $T_{1}$ relaxation times was established as described in the Supplementary Material.

\subsubsection{Relative magnetization}

The relative magnetization of the nuclei of type $i$ at a given location in a flow field that is subjected to a static magnetic field is defined as the actual ratio of the magnetization of that nuclei in the flow field and the maximum magnetization of that nuclei, which would be obtained without flow. The experimental relative magnetization refers to the average value that is measured for the entire active volume of the radio frequency coil. Hence, for a given temperature and component, the relative magnetization of the nuclei of type $i$ only depends on the flow rate. The determination of the relative ${ }^{1} \mathrm{H}$ magnetization was carried out as follows: First, the flow setup was filled with the fluid. Mixtures were prepared gravimetrically using a analytical balance (XS603S DeltaRange, Mettler Toledo, accuracy: $\pm 10 \mathrm{mg}$ ). The mole fractions of acetonitrile $x_{\mathrm{ACN}}$ in the mixtures were $0.3 \mathrm{~mol} \mathrm{~mol}^{-1}$ and $0.8 \mathrm{~mol} \mathrm{~mol}{ }^{-1}$, respectively. The ${ }^{1} \mathrm{H}$ equilibrium magnetization was mea- 
sured without flow $(\dot{V}=0 \mathrm{ml} / \mathrm{min})$. Then, the flow rate was raised in several steps and the ${ }^{1} \mathrm{H}$ magnetization with flow was obtained. Before NMR measurement the new flow rate was applied for at least 5 min to ensure steady-state. For all measurements the flow direction was bottom-to-top of the NMR spectrometer.

The experimental relative magnetization $M_{\mathrm{rel}, i}^{\mathrm{exp}}$ was calculated from the peak areas $A$ of the nuclei of type $i$ as follows:

$$
M_{\mathrm{rel}, i}^{\exp }=\frac{A_{i}(\dot{V})}{A_{i}(\dot{V}=0)}
$$

The ${ }^{1} \mathrm{H}$ NMR spectra were measured with the following sequence parameters: acquisition time: $6.4 \mathrm{~s}, 32 \mathrm{k}$ data points, one scan, $90^{\circ}$ excitation pulse. Prior to integration, post processing (baseline and phase correction) of the obtained NMR spectra was carried out with the SINC method [31]. MNova software was used for integration. All peaks were integrated without deconvolution as the evaluated peaks were well separated. Also for the case of overlapping peaks, excellent methods are available for quantifying the spectrum, e.g. $[6,31,32]$. The flow influences the peak shape: it may lead to tailing and eventually to peak overlaps. This is only partially accounted for by the present method. As the method aims at quantification, it is not necessary to predict the peak shape fully correctly, only the peak area must be correct. The measurement was repeated at least three times with a sufficient long delay time.

The mole fraction of each component in the mixture was calculated from the peak areas. As the mixtures only contained acetonitrile and water with one signal per component (methyl group in $\mathrm{ACN}$ and $\mathrm{OH}$-group in water, 
respectively) the relation is as follows:

$$
x_{\mathrm{ACN}}^{\exp }=\frac{\frac{A_{\mathrm{CH} 3}}{3}}{\frac{A_{\mathrm{CH} 3}}{3}+\frac{A_{\mathrm{OH}}}{2}}
$$

The absolute error of this quantitative evaluation was checked previously with reference samples and is about $0.005 \mathrm{~mol} / \mathrm{mol}$.

\section{Modelling and simulation}

\subsection{Transport model for magnetization}

The simulations of the flow field were done by CFD, i.e. the NavierStokes equations were solved numerically to determine the three dimensional velocity field $\vec{u}(x, y, z)$ in the flow cell assuming a laminar flow and constant temperature and liquid density. It was shown that these assumptions are reasonable for all flow cells. More information is given in the Supplementary Material.

Based on the results for the flow field and information on the external magnetic field, the field of magnetization in the flow cell was calculated from the Bloch-equations [33] based on a transport model, which is described in the following. The simple superposition is possible as the velocity field is not affected by the magnetic field.

When a sample at rest is placed in a constant magnetic field $B_{0, z}$ that acts in direction $z$, the longitudinal magnetization of the nuclei of type $i, M_{i, z}$, changes as a function of time as described by the reduced Bloch-equations in Equation 3.

$$
\frac{\mathrm{d} M_{i, z}}{\mathrm{~d} t}=-\frac{M_{i, z}-M_{0, i, z}}{T_{1, i}}
$$


$T_{1, i}$ is the longitudinal relaxation time of the nuclei of type $i$ and $M_{0, i, z}$ is the equilibrium magnetization of $i$ (i.e. the number of $M_{i, z}$ for $t \rightarrow \infty$ ) which is related to the magnitude of the external magnetic field $B_{0, z}$ by

$$
M_{0, i, z}=\frac{\rho_{0, i} \gamma^{2} \hbar^{2}}{4 k T} \cdot B_{0, z}
$$

where $\rho_{0, i}$ is the spin density of $i, \gamma$ the gyromagnetic ratio, $\hbar$ the reduced Planck constant, $k$ the Boltzmann constant and $T$ the absolute temperature. Due to the variation of $B_{0, z}$ with the flow direction $x$ (cf. Figure 1), $M_{0, i, z}$ and $M_{i, z}$ are a function of the position $x$. As the magnetic field only acts in direction $z, B_{0, z}, M_{0, i, z}$, and $M_{i, z}$ are handled as scalar values.

To describe the magnetization $M_{i, z}$ in a flow field, Equation 3 has to be modified to account for the diffusive and convective transport of the magnetization. The resulting partial differential equation is shown in Equation 5.

$$
\frac{\partial M_{i, z}}{\partial t}+\nabla \cdot\left(\vec{u} M_{i, z}\right)-\nabla \cdot\left(D_{\mathrm{M}, j(i)} \nabla M_{i, z}\right)=-\frac{M_{i, z}-M_{0, i, z}}{T_{1, i}}
$$

The terms on the left hand side of Equation 5 describe the accumulation, the convective and the diffusive transport of the magnetization $M_{i, z}$. The term on the right hand side represents the source term of the magnetization caused by the static magnetic field $B_{0, z} . D_{\mathrm{M}, j(i)}$ is the self-diffusion coefficient of the molecule $j$ to which nuclei of type $i$ belong.

\subsection{Simulation}

The CFD simulations were performed with an augmented version of the $\mathrm{C}++$ open source code OpenFoam 2.4.0. The Navier-Stokes equations were discretized with the finite-volume method (FVM). The computational meshes consisted of hexagonal mesh elements. In the area of expansion in flow cells 
II and III the mesh was refined in order to accurately calculate the changing flow profiles. For flow cell I the number of mesh elements was about 2 millions, for flow cell II and III it was about 10 millions (cf. Figure 1). The discretization of the cylindrically shaped flow cells by hexagonal mesh elements led to small differences between the volume of the flow cell in the simulation and the experiment. Therefore, the flow rate of the simulation was corrected such that mean velocity of simulation and experiment were equal. The correction was less than $1 \%$. It was checked previously that the chosen mesh and the solver options of the CFD simulation give plausible and accurate solutions. Details are given in the Supplementary Material.

The magnetization $M_{i, z}$ was calculated in two steps. As the velocity field is not affected by the magnetic field, first, $\vec{u}$ was predicted by solving the Navier-Stokes equations in the simpleFoam solver [34]. It was assumed that the fluid is Newtonian and the influence of gravity was neglected. In the second step, the solution for $\vec{u}$ was handed over to the BlochFoam solver in which the transport equation for the magnetization (Equation 5) was solved. The source code is supplied electronically in the Supplementary Material.

The Navier-Stokes equations were solved using experimental values from the present work for the density. The numbers for the dynamic viscosity were taken from Wode [35] and those for the self-diffusion coefficients from Hawlicka [36]. Experimental values of $T_{1}$ determined in the present work were used. The scalar field of equilibrium magnetization $M_{0, i, z}$ was calculated from experimental values of the magnetic field strength $B_{0, z}$ using Equation 4. At the inlet of the simulated part of the flow cell ( $x=0$, cf. Figure 1) the flow profile was assumed to be laminar and the magnetization $M_{i, z}$ was set to 
zero. As convergence criteria for pressure and velocity a relative residual of $10^{-6}$ was used. For the magnetization that number was $10^{-9}$.

The predicted scalar field of magnetization $M_{i, z}$ was averaged over planar bins orthogonal to flow direction. The predicted relative magnetization $M_{\mathrm{rel}, i}^{\mathrm{sim}}$ was then determined as follows:

$$
M_{\mathrm{rel}, i}^{\mathrm{sim}}(x, \dot{V})=\frac{M_{i, z}(x, \dot{V})}{M_{0, i, z, \max }}
$$

For the comparison with the experimental relative magnetization, the numbers for $M_{\mathrm{rel}, i}^{\operatorname{sim}}(x, \dot{V})$ were averaged over the active volume of the radio frequency coil. To account for the non-uniform excitation profile of the nuclei by the pulse in the radio frequency coil a weighing function was applied on $M_{\mathrm{rel}, i}^{\mathrm{sim}}$ (see Supplementary Material for more details).

The predicted relative magnetization $M_{\mathrm{rel}, i}^{\mathrm{sim}}$ was used for correcting the experimental mole fraction of acetonitrile as follows:

$$
x_{\mathrm{ACN}}^{\mathrm{cor}}=\frac{\frac{A_{\mathrm{CH} 3}}{3 \cdot M_{\mathrm{rel}, \mathrm{ACN}}^{\mathrm{sim}}}}{\frac{A_{\mathrm{CH} 3}}{3 \cdot M_{\mathrm{rel}, \mathrm{ACN}}^{\mathrm{sim}}}+\frac{A_{\mathrm{OH}}}{2 \cdot M_{\mathrm{rel}, \mathrm{OH}}^{\mathrm{sim}}}}
$$

The relative error of the mole fraction of acetonitrile $\Delta x_{\mathrm{ACN}}^{\mathrm{rel}}$ was calculated using the gravimetrically determined number $x_{\mathrm{ACN}}^{\mathrm{grav}}$ as a reference:

$$
\Delta x_{\mathrm{ACN}}^{\mathrm{rel}}=\frac{x_{\mathrm{ACN}}^{\mathrm{grav}}-x_{\mathrm{ACN}}}{x_{\mathrm{ACN}}^{\mathrm{grav}}}
$$

\section{Results and discussion}

In the present section, the effect of the flow rate on the magnetization in the three flow cells is discussed using simulation and experimental data. The results that were obtained for pure water and for mixtures of water and 
acetonitrile are presented. Those for pure acetonitrile, for which all trends are similar to those obtained for water, are presented in the Supplementary Material. The numerical experimental values of $T_{1}$ as a function of composition and the numerical experimental and simulation values of $M_{\mathrm{rel}, i}$ as a function of flow rate and composition are reported in the Supplementary Material.

\subsection{Pure water}

Figure 2 shows color-maps of the predicted relative magnetization of water flowing at different flow rates through flow cell I. The relative magnetization of water is indicated by the color code. Results for different flow rates ranging from 0.15 to $4 \mathrm{ml} / \mathrm{min}$ as well as for the equilibrium magnetization are shown.

The radial velocity profile (fast in the middle of the flow cell and slow near the wall) has an impact on the magnetization for all flow rates. The lower the velocity, the higher the residence time of the fluid in the magnetic field and the higher the resulting magnetization of the fluid. Hence, the highest magnetization values are reached near the wall. With increasing flow rate more non-magnetized material is transported into the active volume resulting in a lower volume averaged relative magnetization. In an NMR measurement, this reduction of magnetization leads to decreasing signal intensities in the spectrum. The corresponding NMR spectra are shown in Figure 3 for the same flow rates as in the simulation in Figure 2. High flow rates generate a long-range outflow of magnetized material and therefore magnetization is sustained for a longer distance in the flow cell.

Figure 4 shows the predicted relative magnetization of water as a function of the axial position in the flow cell for the studied flow rates in the applied 
flow cells. The equilibrium magnetization $M_{0, z}(\dot{V}=0, t \rightarrow \infty)$ is indicated as dashed line, the shaded area represents the active volume of the radio frequency coil. The magnetization decreases with increasing flow rate. This results in lower values of relative magnetization in the active volume.

In flow cell I (Figure 4a) the magnetization increases monotonously in the direction of flow even for high flow rates indicating that flow is still laminar and no back mixing occurs.

In flow cell II (Figure 4b) the magnetization increases monotonously in the direction of flow only for small flow rates. The section with linear expanding diameter can be clearly seen as the gradient of the magnetization changes. Due to the increasing diameter, the velocity falls and the residence time in the magnetic field increases, which leads to a higher magnetization. At the highest flow rate, for which results are shown in Figure $4 \mathrm{~b}$ a maximum in magnetization occurs. This effect is caused by laminar eddy formation in the section of expansion near the wall, as presented in Figure 5. Due to the back-mixing the fluid is magnetized above the average, which leads to a decrease of magnetization behind the section of expansion. Even though these eddies have a positive effect on the premagnetization of the flowing fluid, they result in a RTD of the fluid that strongly deviates from an ideal flow. This makes the use of such cells for reaction and process monitoring difficult. The influence of the eddy formation on the RTD has to be accounted for. The RTD can be determined either by CFD simulations or experiments.

In flow cell III (Figure 4c) the section of small diameter has almost no contribution to the magnetization because the residence time is to low. Because of the step function of the inner diameter eddies form already at low 
flow rates. The resulting back-mixing causes a large maximum of magnetization behind the expansion. With increasing flow rate the section of enhanced magnetization is broadened because the eddies become enlarged in the direction of flow.

Figure 6 shows a comparison of relative magnetization of water resulting from simulation and experimental data for different flow rates in flow cell I. The experimental data and the predictions show the same trend but there is a slight systematic deviation which results from uncertainties of the input parameters of the simulation. The position of the active volume and the $T_{1}$ time were assumed to be faulty input parameters $( \pm 5 \mathrm{~mm}$ and $\pm 5 \%$ respectively), which is represented by the error bars. As expected, the effect of the uncertainty of the self-diffusion coefficient on the predicted relative magnetization can be neglected. The comparison of simulation and experiment for flow cell II and III gave similar trends to those shown in Figure 6. The results are reported in the Supplementary Material. These comparisons indicate that the model predicts the velocity field, RTD, and the resulting magnetization correctly when the uncertainties of the input parameters are taken into account. This applies for complex geometries and flow patterns.

\subsection{Mixtures of water + acetonitrile}

Figure 7 shows the experimental results of the $T_{1}$ relaxation time of acetonitrile and water protons as a function of the composition in a binary mixture of these components. The variation of $T_{1}$ with composition is not linear. Mixture $T_{1}$ relaxation times differ significantly from those of the pure substances. A possible explanation for this behavior are occurring micro heterogeneity in mixtures of water and acetonitrile [37]. 
Two binary mixtures $\left(x_{\mathrm{ACN}}=0.3\right.$ and $\left.0.8 \mathrm{~mol} \mathrm{~mol}^{-1}\right)$ were investigated in flow cell I and III by simulation and experiment. Figure 8 shows the results of experiment and simulation in flow cell I. For both mixtures the predicted relative magnetization of each proton is in good agreement to the experimental data. The experimental data is slightly underestimated by the model indicating a systematic error in the input parameters of the model. However, the deviation is within the error margin discussed earlier. Both, simulation as well as experimental data confirm the trend of decreasing relative magnetization with increasing flow rate. As follows from Figure 7 , the $T_{1}$ relaxation times of each proton in the investigated mixtures differ by more than $20 \%$. As shown in Section 4.1, this variation of $T_{1}$ has a significant impact on the magnetization of the two species. For both mixtures $T_{1}$ relaxation times of water protons are smaller than those of acetonitrile protons whereby the decrease of relative magnetization is lower for water compared to acetonitrile protons. With the same reason the decrease of relative magnetization is different when comparing one proton species in different mixtures.

Due to the different magnetization of the two species in flow the evaluation of the peak areas gives erroneous mole fractions. To reduce this error the predicted relative magnetization is used as correction factor (cf. Equation 7). A comparison of the relative error in the composition for uncorrected and corrected experimental values in both mixtures is plotted in Figures 8c and 8d. As expected, the error for uncorrected experimental values increases with increasing flow rate because of the lower relative magnetization. The larger the difference in $T_{1}$ relaxation times of each proton in the mixture is, the larger the inherent error of calculated composition becomes. In case of 
mixture $x_{\mathrm{ACN}}=0.3 \mathrm{~mol} \mathrm{~mol}^{-1}$ this results in a relative error up to $29.6 \%$. By means of the model, the error is significantly reduced for both mixtures and for all flow rates (maximum relative error $2.5 \%$ ). This result proves the applicability of the model to use the predicted relative magnetization as correction factor for quantitative NMR measurements of fast flowing liquids.

Figure 9 shows the results of experiment and simulation in flow cell III. For both mixtures the predicted relative magnetization of each proton is in good agreement to the experimental data. The deviations can be explained by the uncertainties of the input parameters. The decrease of relative magnetization with increasing flow rate shows the same trend as already discussed for flow cell I. With higher $T_{1}$ relaxation times the magnetization slows, leading to a lower relative magnetization. This applies for the comparison of water protons with acetonitrile protons (relative magnetization of water is higher than that of acetonitrile) as well as for the comparison of both mixtures. Regarding the relative error in the composition caused by different magnetization of the two species (see Figures $9 \mathrm{c}$ and $9 \mathrm{~d}$ ) the trend from flow cell $\mathrm{I}$ is approved. The error for the uncorrected experimental data increases with higher flow rates and with an increasing difference of $T_{1}$ relaxation times of the protons in mixture. In case of mixture $x_{\mathrm{ACN}}=0.3 \mathrm{~mol} \mathrm{~mol}^{-1}$ this results in a relative error up to $23.1 \%$. By means of the model, the error is significantly reduced for both mixtures and for all flow rates (maximum relative error $-2.2 \%$ ). Again, this result proves the applicability of the model to be used for quantitative NMR measurements of fast flowing liquids in complex geometries. 


\section{Conclusions}

In this work, a CFD-based model was presented that enables quantitative evaluation of NMR measurements of fast flowing liquids in complex flow cell geometries. From the knowledge of the physical properties (density, selfdiffusion coefficient, $T_{1}$ relaxation time) and the flow setup (external magnetic field, flow cell geometry, active volume of radio frequency coil), a prediction of velocity field, residence time distribution, and nuclei and flow rate dependent magnetization is possible for any fluid/flow cell combination. By means of the predicted relative magnetization, a peak area correction of experimental data can be accomplished, that enables to overcome the flow rate limitation induced by the requirements of premagnetization.

For determining the velocity field, the CFD code OpenFoam was used. In a next step, the results were used to calculate the nuclei specific magnetization. The corresponding BlochFoam solver is supplied in the Supplementary Material. To demonstrate the robustness and accuracy of the method the relative magnetization of protons in four liquids (acetonitrile, water, and two mixtures of these components) was predicted in three flow cells (constant inner diameter, linear expanding inner diameter, and step function of inner diameter) at various flow rates. The calculated flow fields helped to understand the observations in the NMR experiments. Even though flow cell II has a continuously increasing diameter, unwanted back mixing occurs at high flow rates. The relative magnetization of both proton species predicted by the model was compared to experimental data. All simulation results were in good agreement to the experimental data, deviations were within the error margins when the uncertainties in the input parameters (position of active 
volume and $T_{1}$ relaxation time) are taken into account.

The predicted relative magnetization was used to correct the peak areas obtained from NMR flow experiments. Without the correction, the relative error in the composition increases with increasing flow rate and with increasing differences in the $T_{1}$ relaxation times of the protons in the mixture, which lead to relative errors in the composition over $25 \%$ in some of the investigated mixtures. By means of the correction the relative error in the composition is reduced to values that are in the range of experimental error (below $2.5 \%$ ).

These results demonstrate that the CFD-based model that was developed in the present work is appropriate to obtain quantitative data from fast flowing NMR measurements in complex flow cell geometries. These results are valid for flow cells with larger inner diameters as well, as the spectrometer is optimized for measurements with standard NMR tubes with outer diameters of $5 \mathrm{~mm}$ and the error caused by field inhomogeneities should be small up to these dimensions. The model can also be used to predict the residence time distribution and relaxation effects of a flowing sample in bypasses or along serial connected analytical devices. Furthermore, the simulation is useful for optimizing the shape and the position of a flow cell in the NMR spectrometer to obtain a maximum of magnetization and avoid back mixing. As the method is predictive, it can be used in a wide range of conditions. In flow cell design and optimization, the time-consuming work with prototypes can be replaced by simulations.

In future work, the method can be extended to flowing hyperpolarized liquids, to elucidate the transport and relaxation of the hyperpolarization. 
Furthermore, the model can be extended to reactive mixtures, for which flow effects are hard to handle using calibration techniques. This can be done by incorporating the corresponding additional exchange terms in the Bloch equation [38].

\section{Acknowledgment}

The authors thank the German Research Foundation (DFG) for the financial support within the Collaborative Research Center SFB/TRR 173 Spin $+\mathrm{X}$. The authors thank Adrian Zoller for his contribution to the experiments of this work. 

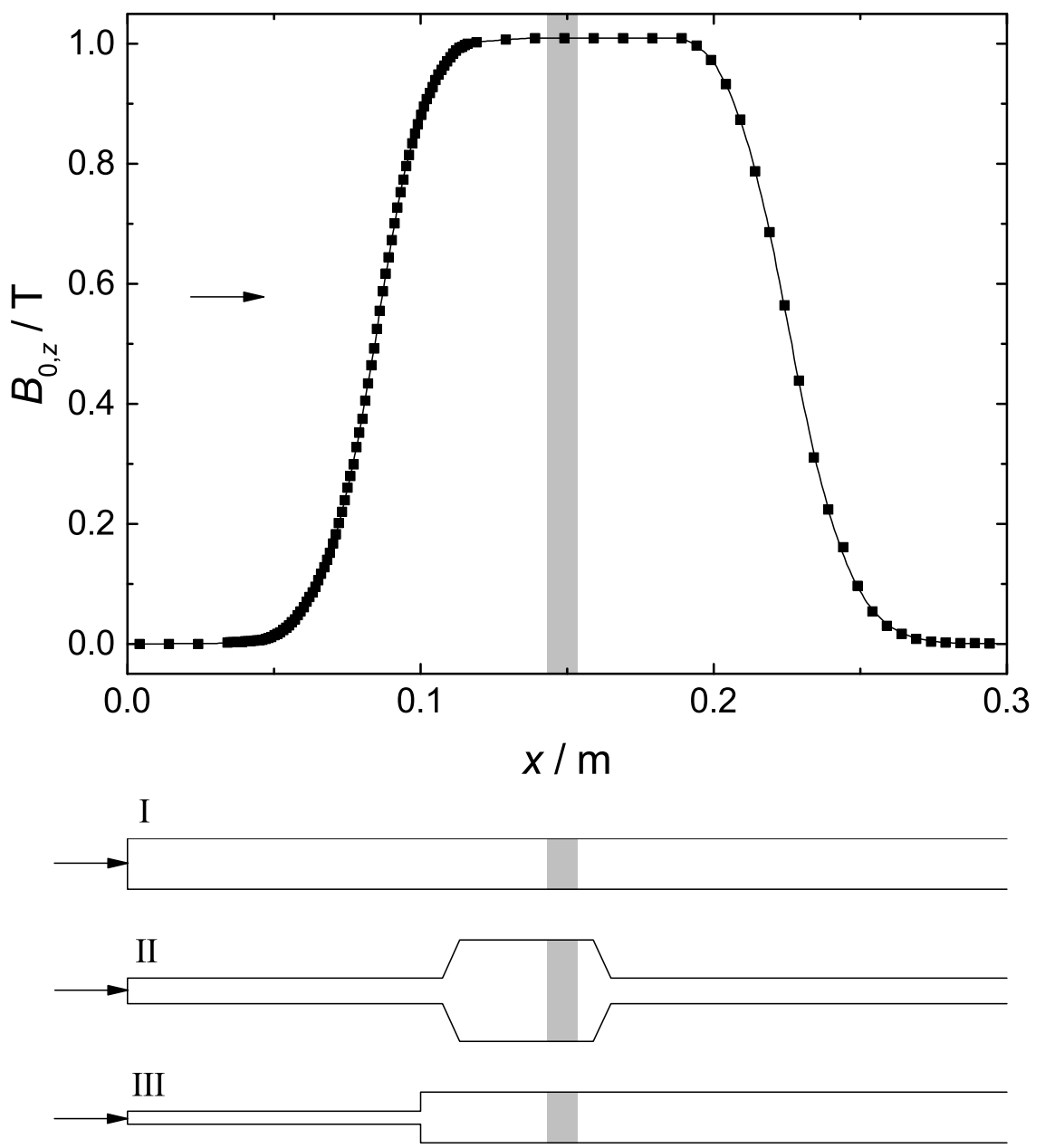

Figure 1: External magnetic field strength and location of the flow cells in the bore of the magnet as a function of the axial position. Symbols: Experimental values; Line: Polynomial fit; Shaded area: Active volume of radio frequency coil. Arrows indicate the direction of the flow (bottom to top). The dimensions of the flow cells are specified in the Supplementary Material. 


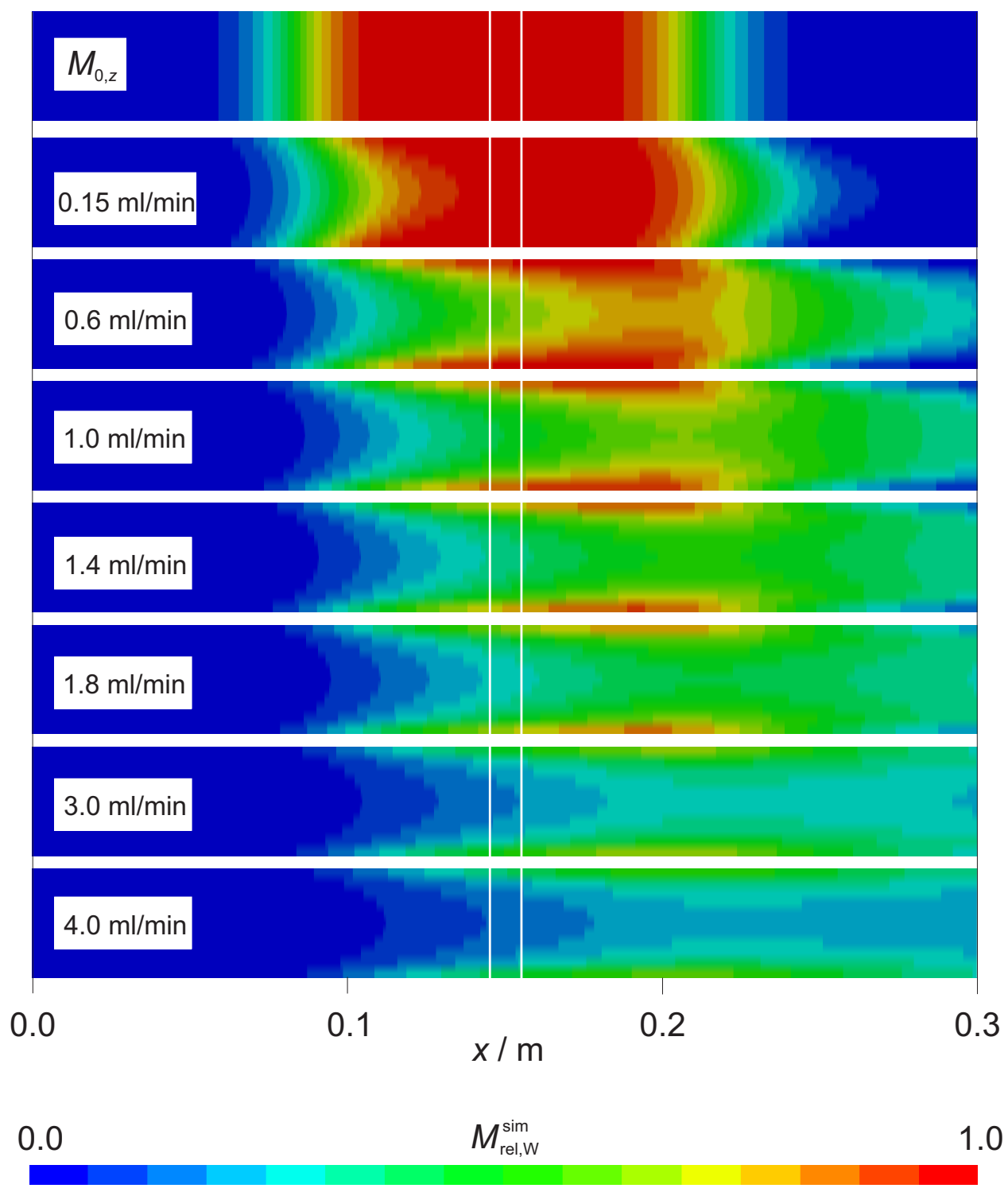

Figure 2: Predicted relative magnetization of water protons in flow cell I at different flow rates. $M_{0, z}$ is the equilibrium magnetization. The white bars indicate the position of the active volume of the radio frequency coil. The corresponding center line velocities are: $v_{\max } / \mathrm{cm} \mathrm{s}^{-1}=0.6,2.5,4.2,5.9,7.6,12.7,17.0$. 


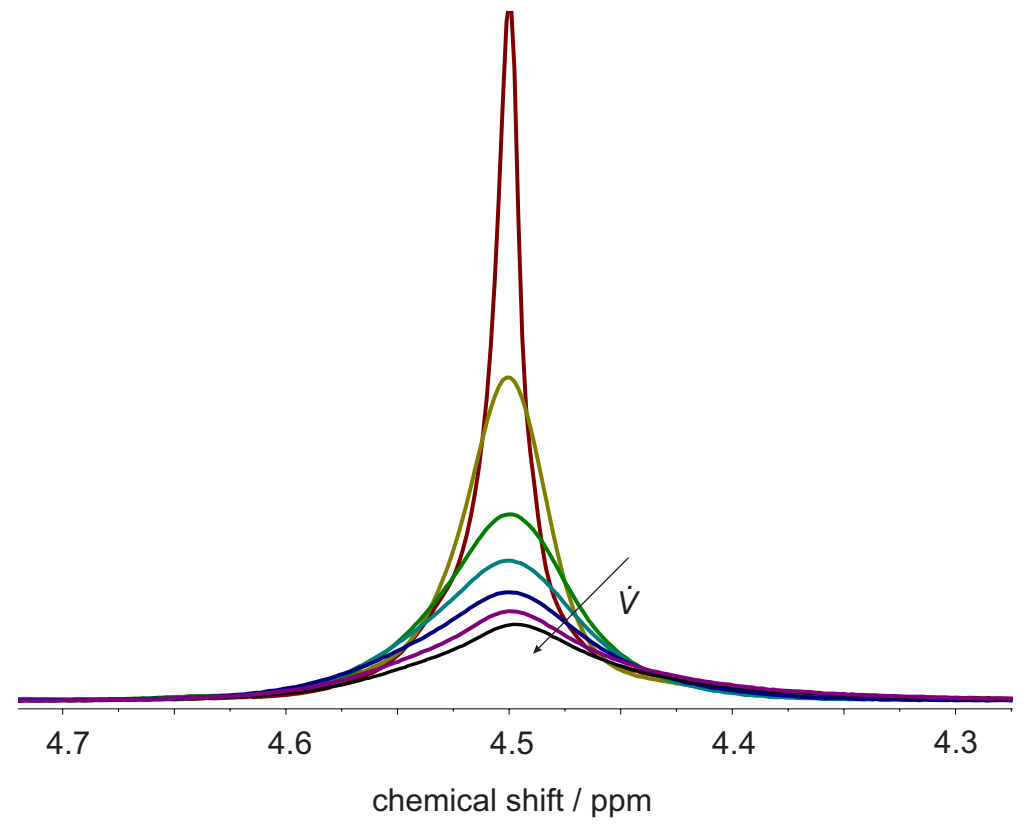

Figure 3: Signal intensity of the water peak in an NMR spectrum for different flow rates $\left(\dot{V} / \mathrm{ml} \mathrm{min}^{-1}=0.15,0.6,1.0,1.4,1.8,3.0,4.0\right)$ in flow cell I (cf. simulations in Figure 2). 
a)

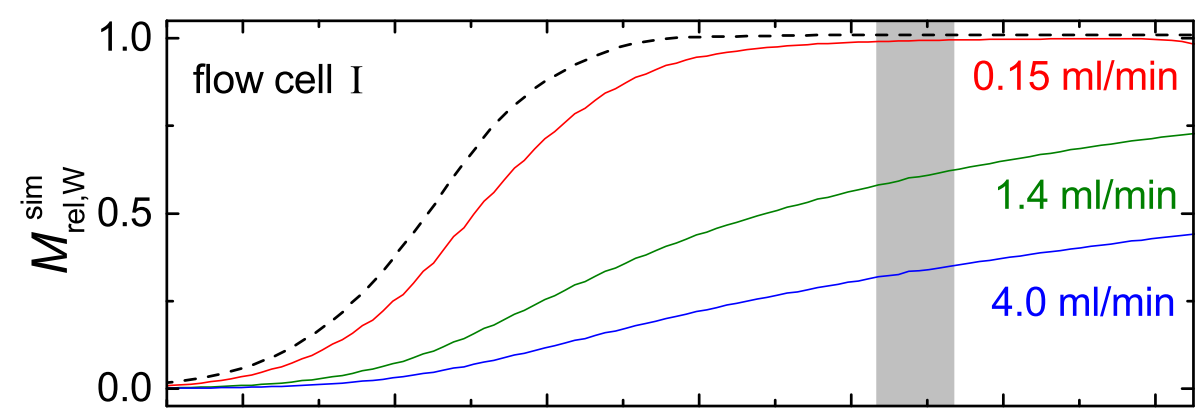

b)

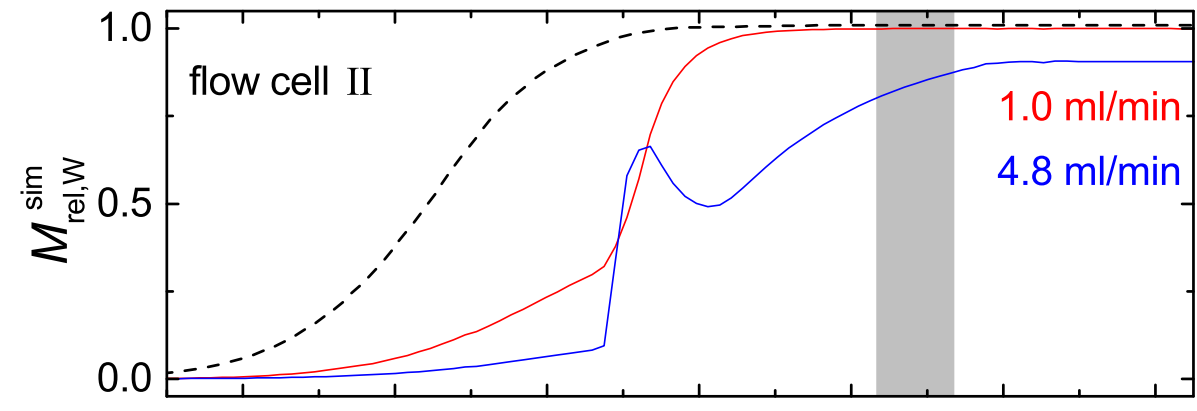

c)

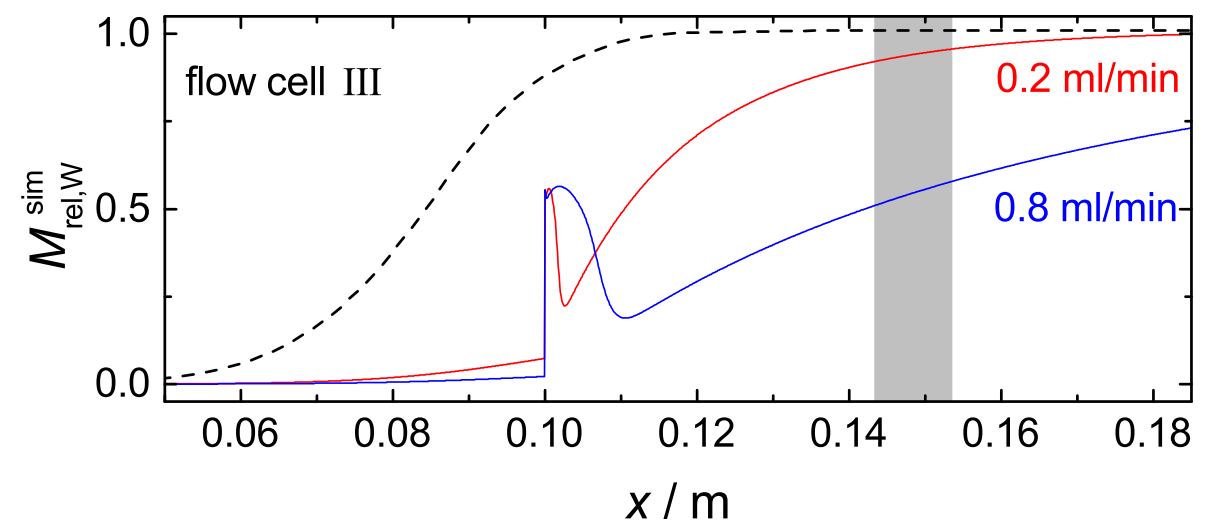

Figure 4: Predicted relative magnetization of water protons for different flow rates as a function of the axial position. a): Flow cell I, $\dot{V}=0.15,1.4$ and $4.0 \mathrm{ml} / \mathrm{min}$; b): Flow cell II, $\dot{V}=1.0$ and $4.8 \mathrm{ml} / \mathrm{min}$; c): Flow cell III, $\dot{V}=0.2$ and $0.8 \mathrm{ml} / \mathrm{min}$; Dashed lines: Equilibrium magnetization; Shaded area: Active volume of radio frequency coil. 


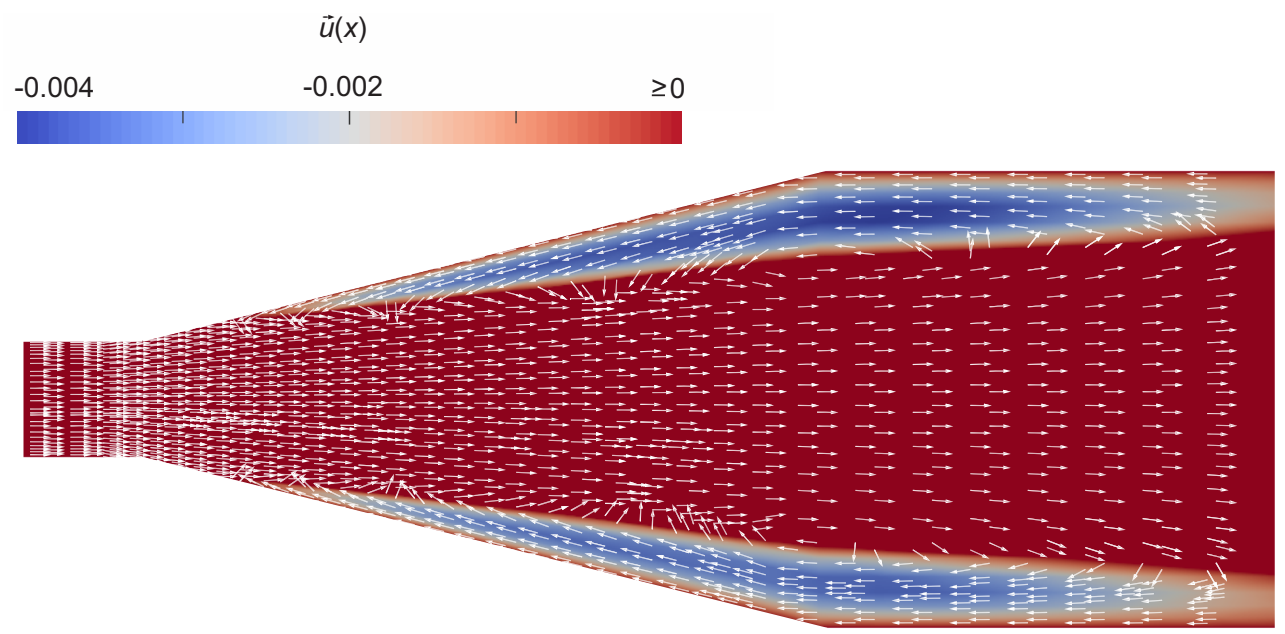

Figure 5: Velocity field in flow cell II for a flow rate of $4.8 \mathrm{ml} / \mathrm{min}$. Colors indicate the velocity values in $x$-direction, for clarity all areas with velocity $\geq 0$ have the same color. Arrows indicate the direction of the flow velocity. 


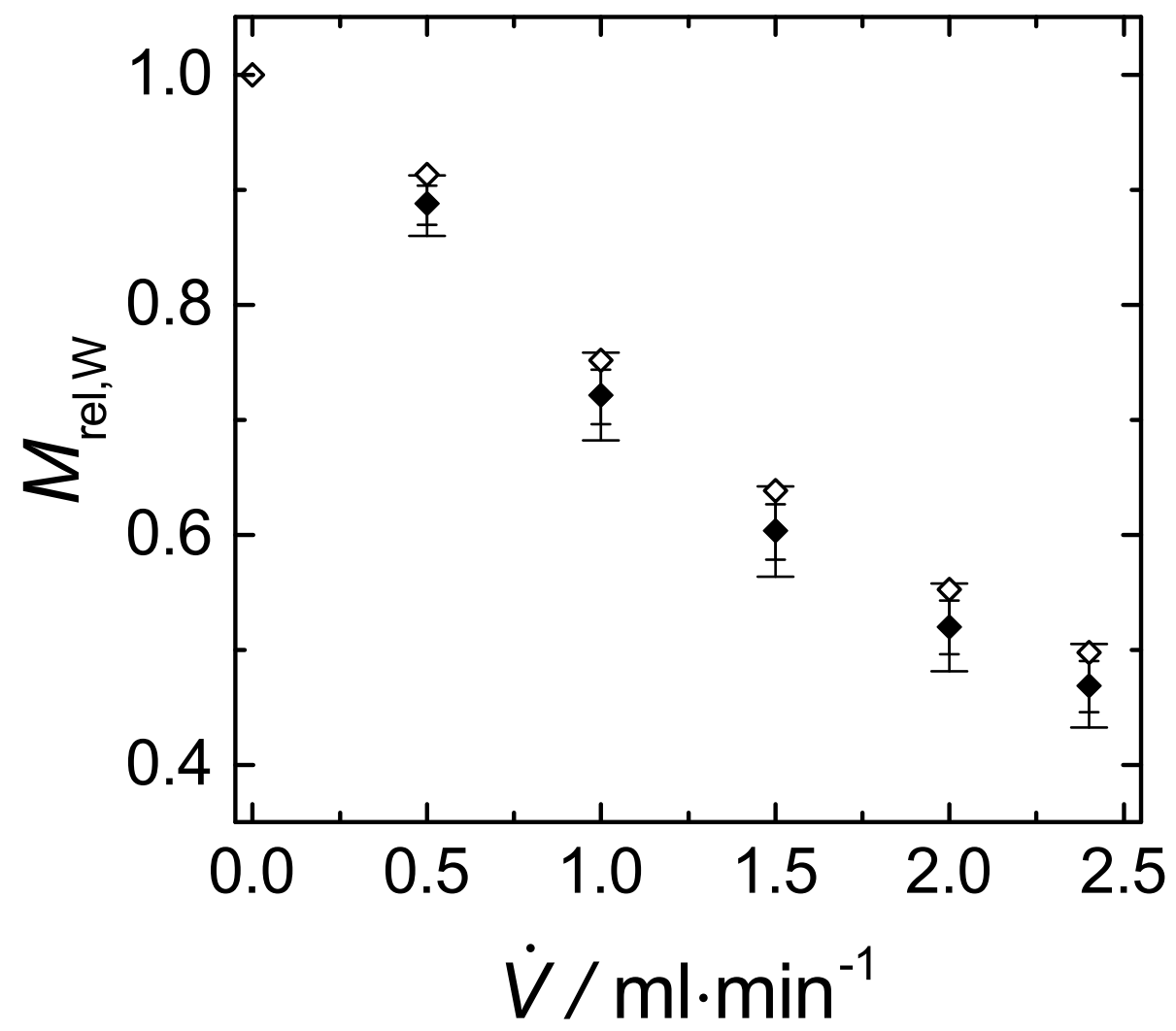

Figure 6: Relative magnetization of water for different flow rates in flow cell I: $(\diamond)$ experiment, $(\bullet)$ model prediction. The error bars of the experimental data points are within symbol size. 


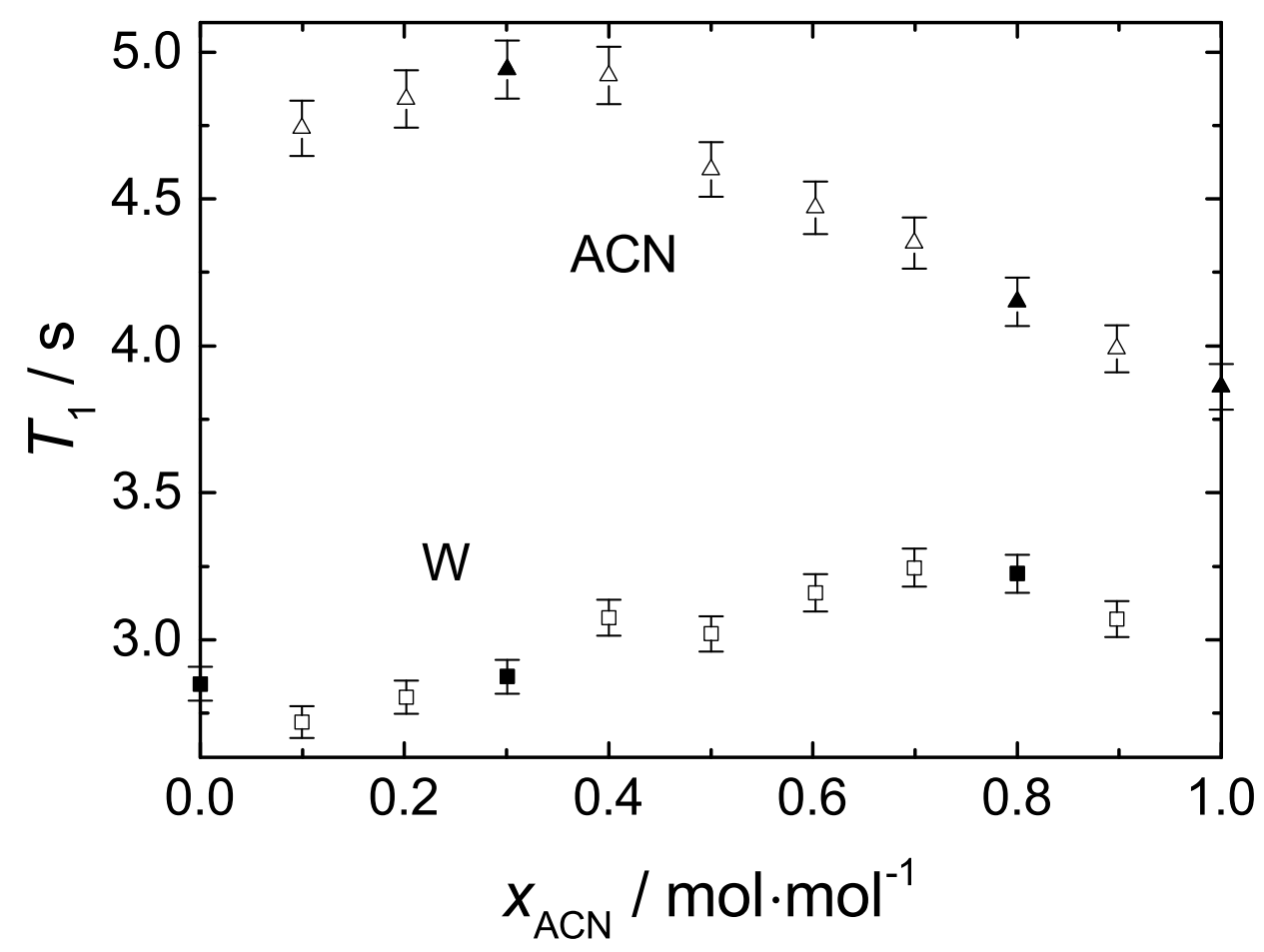

Figure 7: $T_{1}$ relaxation time of acetonitrile and water protons in mixtures of acetonitrile and water at 302 K. $(\triangle)$ acetonitrile, $(\square)$ water. Filled symbols: Investigated mixtures in flow experiments and modeling. 

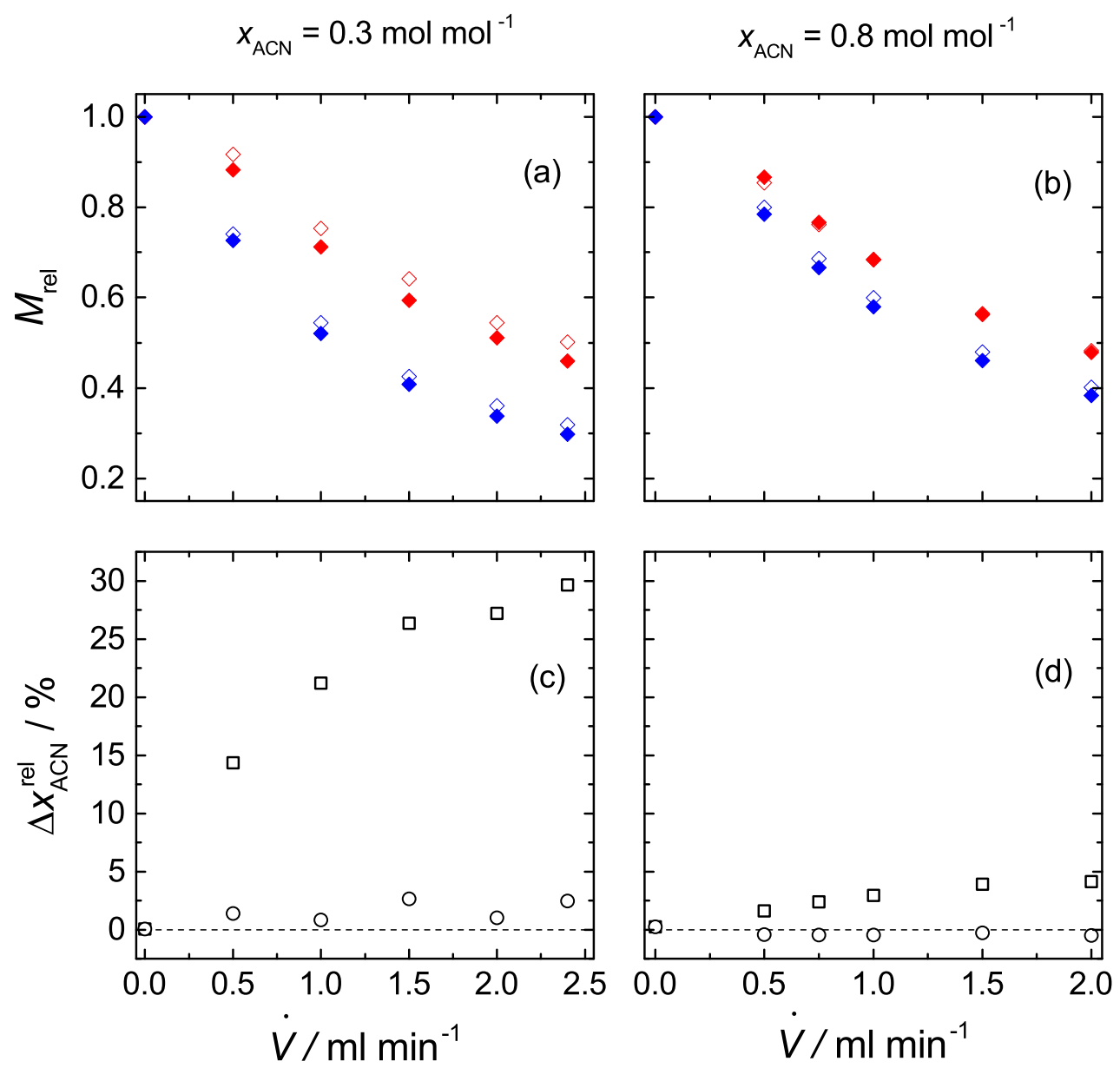

Figure 8: Comparison of simulation and experimental results as a function of flow rate in flow cell I. a) Relative magnetization of protons in mixture $x_{\mathrm{ACN}}=0.3 \mathrm{~mol} \mathrm{~mol}^{-1}$; b) Relative magnetization of protons in mixture $x_{\mathrm{ACN}}=0.8 \mathrm{~mol} \mathrm{~mol}^{-1}$. Red: water, blue: acetonitrile. Open symbols: experiment, filled symbols: model. c) Relative error in the composition in mixture $x_{\mathrm{ACN}}=0.3 \mathrm{~mol} \mathrm{~mol}{ }^{-1}$; d) Relative error in the composition in mixture $x_{\mathrm{ACN}}=0.8 \mathrm{~mol} \mathrm{~mol}^{-1}$. ( $\square$ ) uncorrected, (०) corrected. 

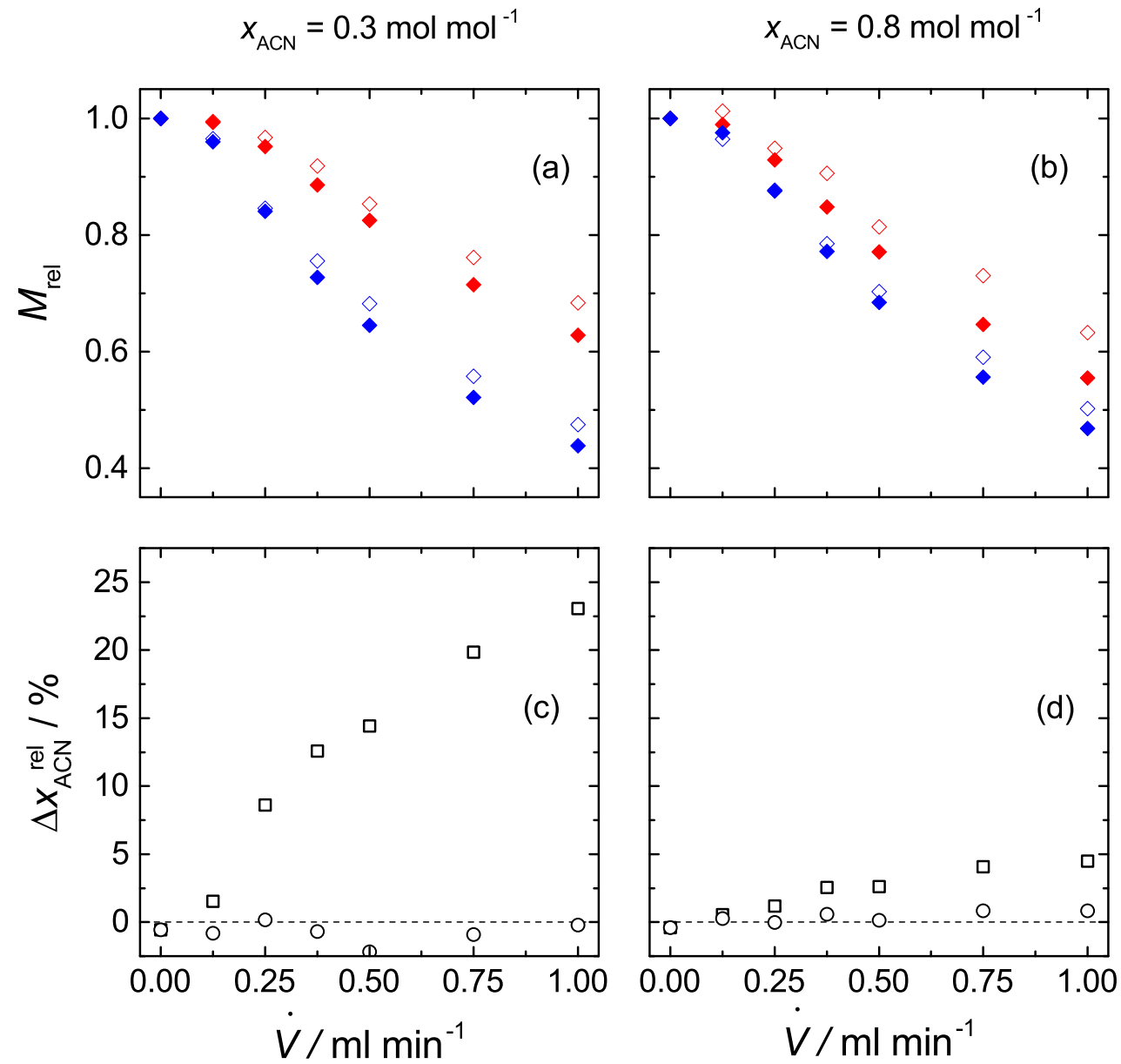

Figure 9: Comparison of simulation and experimental results as a function of flow rate in flow cell III. a) Relative magnetization of protons in mixture $x_{\mathrm{ACN}}=0.3 \mathrm{~mol} \mathrm{~mol}^{-1}$; b) Relative magnetization of protons in mixture $x_{\mathrm{ACN}}=0.8 \mathrm{~mol} \mathrm{~mol}^{-1}$. Red: water, blue: acetonitrile. Open symbols: experiment, filled symbols: model. c) Relative error in the composition in mixture $x_{\mathrm{ACN}}=0.3 \mathrm{~mol} \mathrm{~mol}^{-1} ; \mathrm{d}$ ) Relative error in the composition in mixture $x_{\mathrm{ACN}}=0.8 \mathrm{~mol} \mathrm{~mol}^{-1}$. ( $\square$ ) uncorrected, (o) corrected. 

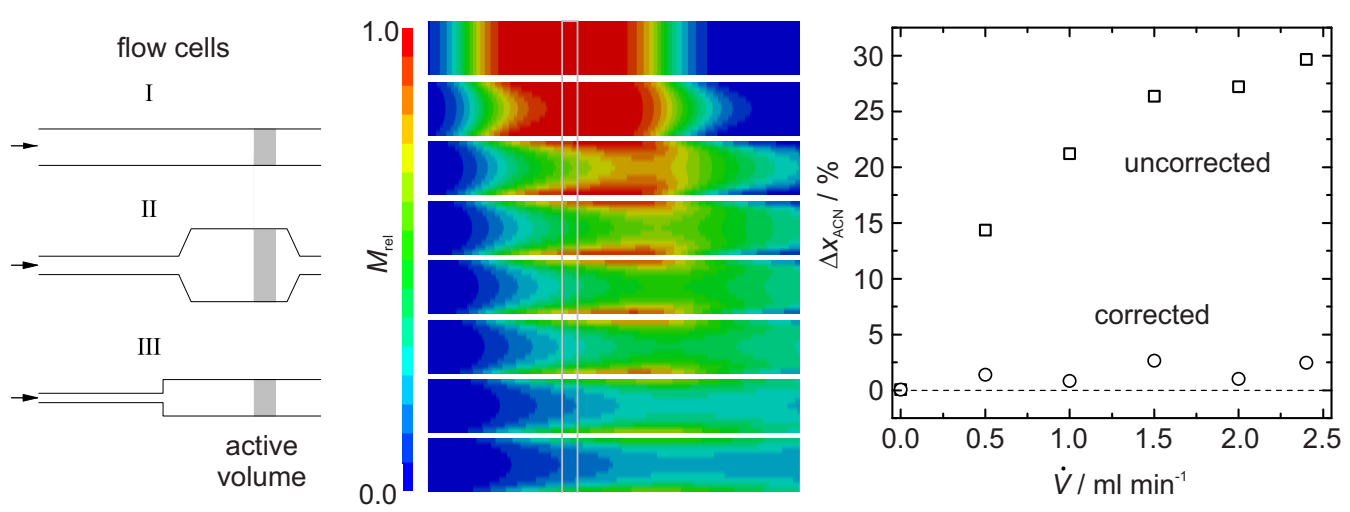

Figure 10: TOC 


\section{References}

[1] M. Maiwald, H. H. Fischer, Y.-K. Kim, K. Albert, H. Hasse, Quantitative high-resolution on-line NMR spectroscopy in reaction and process monitoring, Journal of Magnetic Resonance 166 (2) (2004) 135 - 146. doi:10.1016/j.jmr.2003.09.003.

[2] P. Giraudeau, F.-X. Felpin, Flow reactors integrated with in-line monitoring using benchtop NMR spectroscopy, React. Chem. Eng. 3 (2018) 399-413. doi:10.1039/C8RE00083B.

[3] M. V. Gomez, A. de la Hoz, NMR reaction monitoring in flow synthesis, Beilstein Journal of Organic Chemistry 13 (1) (2017) 285-300. doi:10.3762/bjoc.13.31.

[4] A. Brächer, L. M. Kreußer, S. Qamar, A. Seidel-Morgenstern, E. von Harbou, Application of quantitative inline NMR spectroscopy for investigation of a fixed-bed chromatographic reactor process, Chemical Engineering Journal 336 (2018) 518-530. doi:10.1016/j.cej.2017.12.004.

[5] K. Meyer, S. Kern, N. Zientek, G. Guthausen, M. Maiwald, Process control with compact NMR, TrAC Trends in Analytical Chemistry 83 (2016) 39-52. doi:10.1016/j.trac.2016.03.016.

[6] S. Kern, K. Meyer, S. Guhl, P. Gräßer, A. Paul, R. King, M. Maiwald, Online low-field NMR spectroscopy for process control of an industrial lithiation reaction - automated data analysis, Anal Bioanal Chem 410 (14) (2018) 3349-3360. doi:10.1007/s00216-018-1020-z. 
[7] N. Zientek, C. Laurain, K. Meyer, M. Kraume, G. Guthausen, M. Maiwald, Simultaneous ${ }^{19} \mathrm{~F}^{-1} \mathrm{H}$ medium resolution NMR spectroscopy for online reaction monitoring, Journal of Magnetic Resonance 249 (2014) 53-62. doi:10.1016/j.jmr.2014.10.007.

[8] D. Kreyenschulte, E. Paciok, L. Regestein, B. Blümich, J. Büchs, Online monitoring of fermentation processes via non-invasive low-field NMR, Biotechnol. Bioeng. 112 (9) (2015) 1810-1821. doi:10.1002/bit.25599.

[9] M. V. Gomez, H. H. J.Verputten, A. Diaz-Ortiz, A. Moreno, A. de la Hoz, A. H.Velders, On-line monitoring of a microwave-assisted chemical reaction by nanolitre NMR-spectroscopy, Chemical Communications 46 (25) (2010) 4514-4516. doi:10.1039/B924936B.

[10] S. Bai, B. J. Palmer, C. R. Yonker, Kinetics of deuterium exchange on resorcinol in $\mathrm{D}_{2} \mathrm{O}$ at high pressure and high temperature, J. Phys. Chem. A 104 (1) (2000) 53-58. doi:10.1021/jp991192r.

[11] V. Sans, L. Porwol, V. Dragone, L. Cronin, A self optimizing synthetic organic reactor system using real-time in-line NMR spectroscopy, Chem. Sci. 6 (2) (2015) 1258-1264. doi:10.1039/C4SC03075C.

[12] M. Goldbach, E. Danieli, J. Perlo, B. Kaptein, V. M. Litvinov, B. Blümich, F. Casanova, A. L. L. Duchateau, Preparation of Grignard reagents from magnesium metal under continuous flow conditions and on-line monitoring by NMR spectroscopy, Tetrahedron Letters 57 (1) (2016) 122-125. doi:10.1016/j.tetlet.2015.11.077. 
[13] I. D. Wilson, Multiple hyphenation of liquid chromatography with nuclear magnetic resonance spectroscopy, mass spectrometry and beyond, Journal of Chromatography A 892 (12) (2000) 315-327. doi:10.1016/S0378-4347(00)00071-2.

[14] V. Räntzsch, M. Wilhelm, G. Guthausen, Hyphenated low-field NMR techniques: combining NMR with NIR, GPC/SEC and rheometry, Magn. Reson. Chem. 54 (6) (2016) 494-501. doi:10.1002/mrc.4219.

[15] A. M. R. Hall, J. C. Chouler, A. Codina, P. T. Gierth, J. P. Lowe, U. Hintermair, Practical aspects of real-time reaction monitoring using multi-nuclear high resolution flow NMR spectroscopy, Catal. Sci. Technol. 6 (2016) 8406-8417. doi:10.1039/C6CY01754A.

[16] A. Brächer, S. Hoch, K. Albert, H. Kost, B. Werner, E. von Harbou, H. Hasse, Thermostatted micro-reactor NMR probe head for monitoring fast reactions, Journal of Magnetic Resonance 242 (0) (2014) 155-161. doi:10.1016/j.jmr.2014.02.013.

[17] D. A. Foley, E. Bez, A. Codina, K. L. Colson, M. Fey, R. Krull, D. Piroli, M. T. Zell, B. L. Marquez, NMR flow tube for online NMR reaction monitoring, Anal. Chem. 86 (24) (2014) 12008-12013. doi:10.1021/ac502300q.

[18] E. Danieli, J. Perlo, A. L. L. Duchateau, G. K. M. Verzijl, V. M. Litvinov, B. Blümich, F. Casanova, On-line monitoring of chemical reactions by using benchtop nuclear magnetic res- 
onance spectroscopy, ChemPhysChem 15 (14) (2014) 3060-3066. doi:10.1002/cphc.201402049.

[19] X. Zhang, A. G. Webb, Magnetic resonance microimaging and numerical simulations of velocity fields inside enlarged flow cells used for coupled NMR microseparations, Analytical Chemistry 77 (5) (2005) 1338-1344. doi:10.1021/ac048532b.

[20] R. Haner, Flow tube for NMR probe, Varian Associates Inc 1999, Patent US 5867026 (1997).

[21] A. Nordon, A. Diez-Lazaro, C. W. L. Wong, C. A. McGill, D. Littlejohn, M. Weerasinghe, D. A. Mamman, M. L. Hitchman, J. Wilkie, Consideration of some sampling problems in the on-line analysis of batch processes by low-field NMR spectrometry, Analyst 133 (3) (2008) 339-347. doi:10.1039/B714266H.

[22] J. F. Haw, T. E. Glass, D. W. Hausler, E. Motell, H. C. Dorn, Direct coupling of a liquid chromatograph to a continuous flow hydrogen nuclear magnetic resonance detector for analysis of petroleum and synthetic fuels, Analytical Chemistry 52 (7) (1980) 1135-1140. doi:10.1021/ac50057a032.

[23] A. I. Zhernovoi, G. D. Latyshev, Nuclear magnetic resonance in a flowing liquid, Consultants Bureau, 1965.

[24] M. C. McIvor, A flow probe for nuclear magnetic resonance spectroscopy, Journal of Physics E: Scientific Instruments 2 (3) (1969) 292. 
[25] C. P. Johnston, T. H. West, R. E. Dooley, M. Reid, A. B. Jones, E. J. King, A. G. Leach, G. C. Lloyd-Jones, Anion-initiated trifluoromethylation by TMSCF3: Deconvolution of the siliconatecarbanion dichotomy by stopped-flow NMR/IR, Journal of the American Chemical Society 140 (35) (2018) 11112-11124. doi:10.1021/jacs.8b06777.

[26] D. W. Arnold, L. E. Burkhart, Spinecho NMR response from a flowing sample, Journal of Applied Physics 36 (3) (1965) 870-871. doi:10.1063/1.1714248.

[27] F. Dalitz, L. Kreckel, M. Maiwald, G. Guthausen, Quantitative mediumresolution NMR spectroscopy under non-equilibrium conditions, studied on the example of an esterification reaction, Appl Magn Reson 45 (5) (2014) 411-425. doi:10.1007/s00723-014-0522-x.

[28] F. Dalitz, M. Maiwald, G. Guthausen, Considerations on the design of flow cells in by-pass systems for process analytical applications and its influence on the flow profile using NMR and CFD, Chemical Engineering Science 75 (2012) 318-326. doi:10.1016/j.ces.2012.03.042.

[29] Y. Xia, P. T. Callaghan, K. R. Jeffrey, Imaging velocity profiles: Flow through an abrupt contraction and expansion, AIChE Journal 38 (9) (1992) 1408-1420. doi:10.1002/aic.690380912.

[30] M. Kespe, E. Förster, H. Nirschl, G. Guthausen, Flowing liquids in NMR: Numerical CFD simulation and experimental confirmation of magnetization buildup, Appl Magn Reson 49 (2018) 687-705. doi:10.1007/s00723-018-1016-z. 
[31] M. Sawall, E. von Harbou, A. Moog, R. Behrens, H. Schroeder, J. Simoneau, E. Steimers, K. Neymeyr, Multi-objective optimization for an automated and simultaneous phase and baseline correction of NMR spectral data, Journal of Magnetic Resonance 289 (2018) 132 - 141. doi:10.1016/j.jmr.2018.02.012.

[32] Y. Matviychuk, E. von Harbou, D. Holland, An experimental validation of a bayesian model for quantification in nmr spectroscopy, JMR 285 (2017) 86-100.

[33] F. Bloch, W. W. Hansen, M. Packard, The nuclear induction experiment, Phys. Rev. 70 (1946) 474-485. doi:10.1103/PhysRev.70.474.

[34] S. Patankar, D. Spalding, A calculation procedure for heat, mass and momentum transfer in three-dimensional parabolic flows, International Journal of Heat and Mass Transfer 15 (10) (1972) 1787 - 1806. doi:10.1016/0017-9310(72)90054-3.

[35] H. Wode, Precise measurements of the viscosity of the binary liquid mixtures of acetonitrile - water and 1,3-dimethyl-2-imidazolidinone water., Ph.D. thesis, TU Giessen, 1994.

[36] E. Hawlicka, Acetonitrile-water solutions of sodium halides: Viscosity and self-diffusion of $\mathrm{CH}_{3} \mathrm{CN}$ and $\mathrm{H}_{2} \mathrm{O}$, Z. Naturforsch. 43 (1988) 769773.

[37] J. E. Bertie, Z. Lan, Liquid water-acetonitrile mixtures at $25{ }^{\circ} \mathrm{C}$ : The hydrogen-bonded structure studied through infrared absolute integrated 
absorption intensities, Journal of Physical Chemistry B 101 (20) (1997) 4111-4119.

[38] J.-S. Lee, R. R. Regatte, A. Jerschow, Bloch equations for proton exchange reactions in an aqueous solution, Concepts Magn Reson Part A Bridg Educ Res. 45A (3). 